\title{
Active Virtual Reality Games Reduce Pain Sensitivity in Young, Healthy Adults
}

\author{
Eric Evans ${ }^{1 *}$, Keith E. Naugle ${ }^{2}$, Alex Ovispo ${ }^{3}$, Anthony S. Kaleth ${ }^{2}$, Brent Arnold $^{4}$ and \\ Kelly M. Naugle ${ }^{2}$
}

${ }^{1}$ Department of Health Services Administration, School of Health Professions, University of Alabama at Birmingham, Birmingham, AL, United States, ${ }^{2}$ Department of Kinesiology, School of Health and Human Sciences, Indiana University Purdue University Indianapolis, Indianapolis, IN, United States, ${ }^{3}$ School of Kinesiology, Ball State University, Muncie, IN, United States, ${ }^{4}$ School of Health Sciences, Stockton University, Galloway, NJ, United States

Separately, both physical activity and virtual reality can attenuate pain sensitivity in healthy adults. What is unknown is whether virtual reality combined with physical activity (active virtual reality) could have a greater hypoalgesic effect compared to non-active virtual reality distraction (passive virtual reality engagement).

OPEN ACCESS

Edited by:

Marientina Gotsis,

University of Southern California, Los Angeles, United States

Reviewed by: Frank Steinicke, University of Hamburg, Germany Hai-Ning Liang, Xi'an Jiaotong-Liverpool University, China

*Correspondence: Eric Evans evansej@uab.edu

Specialty section: This article was submitted to Virtual Reality in Medicine, a section of the journal Frontiers in Virtual Reality

Received: 07 September 2021

Accepted: 27 October 2021

Published: 22 November 2021

Citation:

Evans E, Naugle KE, Ovispo A, Kaleth AS, Arnold $B$ and Naugle KM (2021) Active Virtual Reality Games Reduce Pain Sensitivity in Young, Healthy Adults.

Front. Virtual Real. 2:772293. doi: 10.3389/frvir.2021.772293
Objective: The purpose of this study was to determine whether playing physically active virtual reality games exert a greater hypoalgesic effect than a non-active virtual reality game.

Methods: Participants $(n=36)$ played three different active virtual reality games (Beat Saber, Holopoint, and Hot Squat) and one non-active virtual reality game (Relax Walk) for 15 min on four different visits. During gameplay, participants wore accelerometers on the thigh, wrist, and waist to measure movement intensity and quantity. Pressure pain thresholds were measured on the forearm and thigh immediately prior to gameplay (pretest) and immediately following each gaming bout (posttest).

Results: Analysis of the accelerometer data indicated that Hot Squat elicited greater whole-body and lower body moderate to vigorous physical activity compared to the other games. The ANOVA revealed an overall hypoalgesic effect of the virtual reality games on the forearm, regardless of game type. Results also showed a significant hypoalgesic effect on the thigh following gameplay for Hot Squat, Holopoint, and Relax Walk VR. The magnitude of pain reduction was significantly greater during Hot Squat compared to the other games.

Conclusion: Virtual reality gameplay exerted a hypoalgesic effect on experimental pressure pain. Additionally, the data provided evidence of a potential enhanced hypoalgesic effect of physically active virtual reality compared to non-active VR on pressure pain sensitivity.

Keywords: virtual reality, active gaming, physical activity, pressure pain thresholds, pain sensitivity

Abbreviations: BS, beat saber; EIH, exercise induced hypoalgesia; HP, holopoint; HS, hot squat; MVPA, moderate-to-vigorous physical activity; PPT, pressure pain threshold; RW, relax walk VR; VR, virtual reality. 


\section{INTRODUCTION}

Research on virtual reality (VR) as a method of pain reduction has significantly grown in the past 2 decades. This research suggests that passively engaging in VR reduces acute pain sensitivity in healthy and clinical adult populations (Magora et al., 2006; Law et al., 2011; Jin et al., 2016; Glennon et al., 2018). It is suggested that a common mechanism that reduces pain perception in adults during VR primarily relies on distraction, where participants' attention is directed towards the VR environment instead of the pain stimulus (Hayashi et al., 2019). Additionally, distraction could be attributed to the inability of sensory systems to focus on multiple simultaneously active pain stimuli, thereby reducing pain (McCaul and Malott, 1984; Hoffman et al., 2007). For example, in a study examining the effects of VR on ischemic pain, participants reported lower pain levels and spent less time thinking about the induced pain while engaging in a VR environment compared to not undergoing a VR stimulus (Hoffman et al., 2003). Similarly, earlier work in VR and pain has shown positive distraction effects of engaging in VR environments for adults and adolescents who experienced burn pain (Hoffman et al., 2000a; Hoffman et al., 2000b). However, these prior studies on VR's effects on pain have primarily focused on passive engagement (little to no physical activity) or involved short exposure time to VR technology.

Another stimulus that has been shown to produce hypoalgesic effects is physical activity. A large body of evidence indicates that acute bouts of physical activity and exercise produce hypoalgesic effects in healthy adults and chronic pain populations (Naugle et al., 2012; Naugle et al., 2014; Rice et al., 2019). Specifically, research demonstrates that an acute bout of aerobic, isometric, or dynamic strength training exercise can reduce pain sensitivity or perception to experimentally induced pain (Naugle et al., 2012; Rice et al., 2019). This phenomenon is known as exercise-induced hypoalgesia (EIH) (Koltyn, 2000). In healthy, pain-free adults, EIH generally follows an acute bout of exercise that is moderate to vigorous in intensity with a duration of at least $15-20 \mathrm{~min}$ for aerobic exercise and 1-5 min of isometric exercise (Kemppainen et al., 1990; Naugle et al., 2012; Rice et al., 2019).

Separately, both physical activity and VR can attenuate pain in healthy adults. However, little research has investigated whether VR combined with PA (active VR) could have a greater hypoalgesic effect compared to non-active VR distraction (passively engaging in a virtual reality environment with little movement). Recently, Hayashi et al. demonstrated that VR combined with exercise imagery exerted a greater analgesic effect on pressure pain thresholds (PPTs) compared to pure VR distraction (Hayashi et al., 2019). Prior research indicates that exercise imagery increases brain activity in the motor and premotor cortex similar to if actual movements were occurring (Lotze and Halsband, 2006; Miller et al., 2010), and thus may introduce another mechanism (i.e., exercise) through which pain could be decreased. Additionally, prior work by Czub and others provided initial evidence that increased body movements while engaging in head mounted display VR were associated with greater reductions in experimentally induced pain compared to VR without movement (Czub and Piskorz, 2014; Czub and
Piskorz, 2017). However, these movements were localized at the arm level and did not include any significant whole or lower body activity. Overall, the aforementioned studies provided preliminary evidence that perhaps VR-induced hypoalgesia could be enhanced when the distraction effects of VR are combined with hypoalgesic effects of exercise or physical activity (Hayashi et al., 2019).

In recent years, commercial VR systems have released physically active VR games, which allow participants to wear a head-mounted display system and use handheld controllers to interact with a virtually displayed environment through physical movements, using upper body, whole-body, or lower body movements. Little research has investigated the hypoalgesic effects of such active VR games. Therefore, the purpose of this study was to determine whether playing physically active VR games would elicit an acute hypoalgesic effect on experimentally induced pain in young, healthy adults. First, we hypothesized that the VR games would significantly reduce pressure pain sensitivity regardless of the amount of movement following $15 \mathrm{~min}$ of VR gaming. Secondly, we hypothesized that playing VR games which require more physical activity would have a significantly greater hypoalgesic effect than the non-active VR game, via a combined effect of VR distraction and EIH.

\section{MATERIALS AND METHODS}

\section{Participants}

Following approval by the Institutional Review Board, thirty-nine adults between the ages of 18 and 30 were enrolled in this study. This age criteria was chosen due to the higher rates of VR and video game usage among the younger adult population (Weaver et al., 2009). Additionally, older adult populations do not show a consistent hypoalgesic effect from physical activity or exercise (Naugle et al., 2016). Participants were recruited from the Indianapolis area through flyers, word of mouth, and verbal script presentation. Interested participants were instructed to contact the researcher through email to inquire about study eligibility and schedule the first session appointment. All participants were fully informed of the nature of the study and their right to decline participation or withdraw from participation at any point of time. Written informed consent was obtained from all participants. Three participants were unable to complete all study sessions due to events surrounding COVID-19. Only data from participants who completed the entire study was included in the data analysis (36 total).

Inclusion criteria included individuals who were between the ages of 18 and 30 years.

Exclusion criteria for this study included prior or current experiences with motion sickness or claustrophobia, an answer of "Yes" on any of the seven general 2019 Physical Activity Readiness Questionnaire (PAR-Q) items and on the subsequent follow-up questions, and any acute or chronic pain condition. Session exclusion criteria included severe uncontrolled hypertension (resting SBP $>180 \mathrm{mmHg}$, resting DPB $>99 \mathrm{mmHg}$ ), vigorous exercise performed within $12 \mathrm{~h}$ of the session, eating less than 1 hour before the session, smoking or 
TABLE 1 | Virtual reality games descriptions.

\section{Game}

Beat Saber (Active VR)

Holopoint (Active VR)

Hot Squat (Active VR)

Relax Walk VR (Non-active VR)

\section{Description}

Virtual Reality rhythm game where participants use controllers like light sabers that are used to strike incoming objects matched with in-game music

A fast-paced archery game where participants use the controllers as a bow and arrow to hit incoming targets

A squatting-based game where the participant squats up and down to avoid incoming objects

A virtual reality game where participants explore virtual environments by using the controller to transport themselves from place to place. Requires little-to-no upper body movement; can be done sitting or standing
Session 1

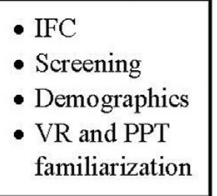

- IFC

reening

- VR and PPT

familiarization

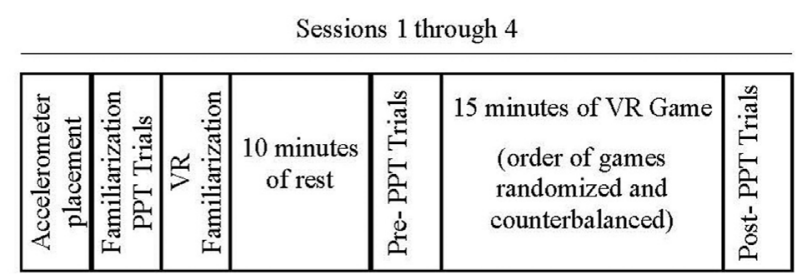

FIGURE 1 | Order of experimental events.

alcohol consumption within $24 \mathrm{~h}$ of scheduled session, caffeine ingested on day of session prior to appointment, analgesic medications taken on the day of the session prior to appointment, and not wearing clothing which allows skin contact for pain testing on the dominant thigh and forearm.

\section{Procedures}

The data reported here, are part of a larger study examining the physical activity levels of active VR games (Evans et al., 2021). Participant enrollment began on January 28, 2020. Participants completed four sessions on separate days in a repeated measures experimental design. The first session included the informed consent process and one experimental game. Sessions 2-4 were devoted to one experimental game per session. All sessions were conducted in the National Institute for Fitness and Sport, located on the IUPUI campus.

\section{Screening and Enrollment (Session 1)}

Participants were given a brief overview of the study procedures and were asked to read and sign an Informed Consent Form (ICF). Following the ICF process, participants were given the PAR-Q and International Physical Activity Questionnaire (IPAQ) to complete. Height, weight, and resting heart rate, and resting blood pressure were collected. Participants were also asked to fill out a demographic questionnaire. Session exclusion criteria were evaluated at the beginning of each experimental session. If session exclusion criteria were not met, then the session was rescheduled.

Familiarization of Pain Test and VR System (Session 1) After study eligibility confirmation, participants underwent familiarization with the pressure pain threshold (PPT) test to measure pain sensitivity (See outcome measure below for description of test). The PPT test was performed as practice on the participants' non-dominant forearm and ipsilateral thigh three times. Following PPT test familiarization, participants were shown the HTC Vive system (HTC, Taiwan; Valve, Washington), which includes a head-mounted display system and two handheld controllers. This VR system uses room-scale tracking technology, which allows the user to move in three-dimensional space and use motion-tracked controllers to translate real-life motion into the VR environment. Two ceiling-mounted base stations mapped the physical space in which the participant played and provided boundaries which informed the user to stay within the designated play area. The base stations also track sensors within the headset and controllers within the virtual environment. The HTC Vive system came with a tutorial program which exposes the user to the basic functions of the VR system. Each participant was fitted with the headset and followed the tutorial for movement and system familiarization.

\section{Experimental Protocol (All Sessions)}

Participants completed four randomized experimental sessions, each separated by at least $24 \mathrm{~h}$. One of the following four VR games was played during each session: Relax Walk (RW: nonactive game), Beat Saber (BS), Holopoint (HP), or Hot Squat (HS). See Table 1 for descriptions of games. The aforementioned games were chosen because each game was reported to elicit different types of physical activity. Specifically, Relax Walk requires little to no movement to play. Beat Saber and Holopoint use primarily upper-body movements during gameplay and Hot Squat uses primarily lower- and wholebody movements. Prior research has shown that different active video games that require more lower-body movement increase overall energy expenditure during gameplay when compared to using only upper extremities (Jordan et al., 2011). The amount and type of movement of each game was verified with accelerometers during gameplay, as described below. Four counterbalanced orders of the games were generated, and each participant was randomly assigned to one of the four game 
orders, with nine participants per order. Following familiarization via the tutorial (only Session 1), participants were fitted with three accelerometers worn on the dominant wrist, the dominant hip in line with the armpit, and the ipsilateral thigh just above the knee. Then, participants were introduced to one of the games through a verbal description by the researcher and were allowed to play the game for $5 \mathrm{~min}$ for familiarization. Afterwards, the participants stopped playing and sat in a resting position to return to resting heart rate. After $10 \mathrm{~min}$ of rest, the participant played the VR game for $15 \mathrm{~min}$. Participants were instructed to play the VR game at a self-selected pace. PPT testing was performed three times during the experimental session: 1) prior to VR familiarization (familiarization trials), 2) immediately before the $15 \mathrm{~min}$ VR game play (pretest), and 3) immediately after VR game play (posttest). See Figure $\mathbf{1}$ for an overview of the order of events.

\section{Outcome Measures \\ Pressure Pain Thresholds}

A digital, handheld, clinical grade pressure algometer with a $1 \mathrm{~cm}$ rubber tip (Wagner Instruments, Greenwich, CT) was used to assess PPTs on the forearm and thigh. The experimenter applied a slow constant rate of pressure to the skin surface, with a corresponding number on the device indicating the pressure amount. Pressure was applied until the first sensation of pain was signaled by the participant, after which the algometer was immediately removed. Pressure pain threshold was defined as the amount of pressure in foot-pounds at which the participant first reported experiencing pain. Two trials were performed at each body site (4 trials total at each time point) during each time point. The specific body sites included the anterior dominant arm $8 \mathrm{~cm}$ down from the participants elbow crease and dominant thigh at $10 \mathrm{~cm}$ above the knee. Inter-trial-intervals were $20 \mathrm{~s}$. The order of the PPT's at each body site (forearm and thigh) was randomized and counterbalanced across participants to reduce an order effect. The PPT trials were completed after resting measures (before game familiarization), immediately before the $15 \mathrm{~min}$ VR gameplay, and immediately after the $15 \mathrm{~min}$ VR gaming session while the participant was still wearing the VR headset. Pressure pain threshold testing is a reliable method of assessing pressure pain in healthy, young adults (Chesterton et al., 2007; Aytar et al., 2014; Bisset et al., 2015; Waller et al., 2015). Percent change in PPTs was calculated from Pre-VR to Post-VR to evaluate the magnitude of hypoalgesic differences among the VR games using the following method [(Post-VR PPT -PreVR $\left.\mathrm{PPT}) /(\text { Pre-VR PPT })^{\star} 100\right]$. The average value from the two trials for each site and each time point were used in statistical analysis.

\section{Accelerometry}

ActiGraph GT3X + accelerometers (The ActiGraph Inc. Pensacola, FL) were worn on the dominant wrist, ipsilateral hip, and ipsilateral thigh during all sessions of VR play. The ActiGraph is a small lightweight tri-axial accelerometer that is designed to detect tri-axial accelerations in the range of $0.05-2 \mathrm{G}$. Output from the ActiGraph was in the form of step counts, body positions and activity counts for a specific time period. Data was captured in $1 \mathrm{~s}$ epochs. The accelerometer data used for analyses
TABLE 2 | Descriptive statistics of sample (Means and SD).

\begin{tabular}{lcc}
\hline & Male & Female \\
\hline Age (years) & $22.2(2.8)$ & $21.1(3.1)$ \\
IPAQ (MET*minutes/week) & $6188.92(4938.06)$ & $4140.11(4399.48)$ \\
BMl (kg/m $\left.{ }^{2}\right)$ & 28.39 & 23.53 \\
VR Experience (\%) & & \\
$\quad$ No experience & $83 \%$ & $89 \%$ \\
$\leq 1$ time per week & $17 \%$ & $11 \%$
\end{tabular}

$S D$, standard deviation; $m$, meter; kg, kilogram; IPAQ, international physical activity questionnaire; BMl, body mass index.

was calculated from minutes 2 through 14 (13 min total) of each 15 min active gaming period to represent steady-state activity. The ActiLife software (Pensacola, FL) was used to process the Actigraph data, with the "worn on wrist" correction applied for the wrist accelerometer. Activity count cut-points (e.g., counts/ min) were used to determine the amount of time a subject spent in sedentary time $(<100)$ and moderate-to-vigorous physical activity (MVPA: >1951) (Freedson et al., 1998). The Actigraph GT3X+ is a valid and reliable tool and has been used in prior active gaming studies to measure physical activity intensity levels (Kelly et al., 2013; Aadland and Ylvisåker, 2015; Kim et al., 2015; Jones et al., 2018; Naugle et al., 2019).

\section{Data Analysis \\ Power Analysis}

A power analysis was conducted using G Power 3.1.5 to determine an appropriate sample size. A meta-analysis of exercise induced hypoalgesia revealed a moderate to large effect of acute exercise on experimental pain (Naugle et al., 2012). Additionally, prior research has shown moderate to large effects of VR on experimentally induced pain (Demeter et al., 2015) Thus, we conducted our power analysis to detect a moderate effect size. Our power analysis showed that the minimum sample size for detecting within-group differences with a moderate effect size $(f=0.25)$ between the pre- and post-pain measures with an alpha level of 0.05 and power of 0.80 was sixteen (Lotze and Halsband, 2006) subjects.

\section{Statistical Software and Analysis}

SPSS was used for data analysis. Means and standard deviations for each variable and for each condition were calculated. Descriptive statistics for demographic variables and IPAQ data were also calculated. Repeated measures ANOVAs were conducted on sedentary minutes, and MVPA minutes at each body site to determine differences in each type of physical activity across the games.

We conducted a preliminary analysis to determine whether the PPTs significantly changed from the familiarization assessment to the pre-test using a 4(Game) $\mathrm{x}$ 2(Time: familiarization vs. pretest) x 2 (sex) mixed model ANOVA. The results showed that PPTs did not significantly change from familiarization trials to pretest trials for the forearm PPT $(p<0.922)$ and thigh PPT $(p<0.193)$. Therefore, the primary focus was on differences between pre-VR PPT and post-VR PPT data. Thus, our main hypothesis was evaluated with 4 (Game) $\mathrm{x} 2$ 
TABLE 3 | Means and standard deviations (SD) for Pressure Pain Thresholds across Game, Time, and Body Site.

\begin{tabular}{lcccc} 
& Beat Saber & Holopoint & Hot Squat & Relax Walk VR \\
\hline Thigh & & & & \\
$\quad$ Familiarization & $13.0(8.1)$ & $12.6(8.1)$ & $14.0(10.1)$ & $13.3(9.1)$ \\
$\quad$ Pre-VR & $13.5(9.7)$ & $12.6(8.8)$ & $14.2(9.4)$ & $13.5(9.7)$ \\
$\quad$ Post-VR & $14.1(9.8)$ & $14.2(10.3)$ & $16.3(10.7)$ & $14.7(11.4)$ \\
Forearm & & & & \\
$\quad$ Familiarization & $9.2(8.3)$ & $8.7(7.9)$ & $9.2(6.8)$ & $9.6(8.7)$ \\
$\quad$ Pre-VR & $9.5(8.5)$ & $8.6(7.1)$ & $9.2(7.5)$ & $9.5(7.7)$ \\
$\quad$ Post-VR & $10.0(8.7)$ & $9.2(7.9)$ & $9.9(7.2)$ & $9.8(9.0)$ \\
\hline
\end{tabular}

Note: VR, virtual reality; Pain Pressure Threshold units: Ib*ft.

(Time: pretest vs. posttest) x 2 (sex) mixed model ANOVAs. These analyses were conducted separately for the average PPTs on the forearm and thigh. We also evaluated the PPT percent change scores with 4 (Game) x 2 (Sex) mixed model ANOVAs. These analyses were used to determine the magnitude of hypoalgesic differences between games. Similarly, these analyses were conducted separately for the forearm and thigh. If the sphericity assumption was violated, then GreenhouseGeisser degrees of freedom corrections was applied to obtain the critical $p$-value. Post-hoc analyses were conducted by using the Tukey HSD test. Statistical significance was determined at $p \leq$ 0.05 for all analyses.

\section{RESULTS}

\section{Descriptive Characteristics}

Thirty-six $(n=36)$ participants completed all sessions, with equal number of males and females. Sample characteristics include age, body mass index (BMI), and IPAQ scores (Table 2). The Mann-Whitney $U$ test showed that the IPAQ scores were not significantly different between males and females $(p=0.161)$. Age was not significantly different between males and females $(p=0.272)$, while BMI was significantly different between males and females $(p=0.018)$ with males having higher BMI's than females. Scores from the IPAQ were compared to the categories established by the IPAQ data processing method (Craig et al., 2003). Total IPAQ scores show that the study sample fell within the High $(>3000$ $\mathrm{MET}^{\star}$ minutes/week) physical activity category. All participants reported low to no experience with VR gaming.

\section{Hypoalgesic Effects of VR Games \\ Pressure Pain Thresholds}

The ANOVA conducted on forearm PPTs showed a main effect of time $(p<0.004)$, with a significant increase from Pre-VR PPTs $\left(9.16 \pm 7.01 \mathrm{lb}^{\star} \mathrm{ft}\right)$ to Post-VR PPTs $\left(9.72 \pm 7.48 \mathrm{lb}^{\star} \mathrm{ft}\right)$. The ANOVA for the forearm PPTs also showed a main effect of sex $(p<0.022)$. This main effect was superseded by a game by sex interaction $(p<0.023)$. Follow-up Tukey HSD showed that the forearm PPTs for males (Beat Saber $=12.81 \pm 8.01 \mathrm{lb}^{\star} \mathrm{ft}$; Holopoint $=10.85 \pm 7.29 \mathrm{lb} \mathrm{ft}^{*}$ Hot Squat $=12.71 \pm 6.63$ $\mathrm{lb}^{\star} \mathrm{ft}$; Relax Walk $=12.94 \pm 7.75 \mathrm{lb} \mathrm{ft}^{\star}$ ) was significantly higher than females (Beat Saber $=6.60 \pm 8.01 \mathrm{lb}^{\star} \mathrm{ft}$; Holopoint $=6.89 \pm$ $7.29 \mathrm{lb}{ }^{\star} \mathrm{ft}$; Hot Squat $=6.38 \pm 6.63 \mathrm{lb}{ }^{\star} \mathrm{ft}$; Relax Walk $=6.35 \pm 7.75$ $\mathrm{lb}^{\star} \mathrm{ft}$ ) across all games. Additionally, male forearm PPTs during Beat Saber, Hot Squat, and Relax Walk was significantly higher than during Holopoint. All other effects and interactions including game $(p<0.269)$, time by sex $(p<0.362)$, game by time $(p<0.855)$, and game by time by sex $(p<0.442)$ were not statistically significant.

The ANOVA conducted on thigh PPTs also showed a main effect of time $(p<0.001)$. This main effect was superseded by a game by time interaction $(p<0.010)$. Follow-up Tukey HSD tests showed that thigh PPT values significantly increased from preVR to post-VR for Holopoint, Hot Squat, and Relax Walk, but not for Beat Saber. Means and standard deviations for PPT's for each game by time and body site are in Table 3 .

\section{Percent change in PPTs}

We also evaluated the percent change in PPTs for the forearm and thigh. The ANOVA revealed no main effects for game $(p<0.372)$ and sex $(p<0.886)$, and no significant game by sex interaction $(p<0.328)$ on percent change in forearm PPT's. Thus, the magnitude of hypoalgesia at the forearm did not differ significantly between games. For the thigh PPT's, the ANOVA showed a main effect of game $(p<0.013)$, no effect of sex $(p<$ $0.739)$ and no game by sex interaction $(p<0.479)$. Follow-up Tukey HSD tests on the main effect of game showed that percent

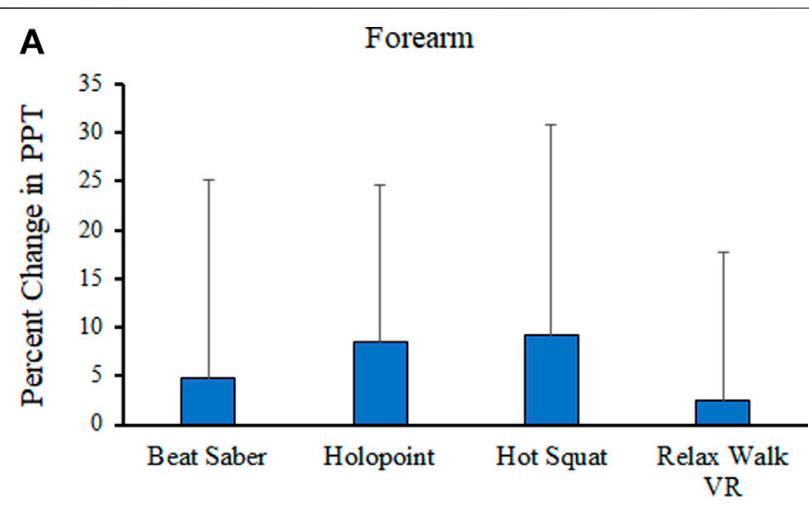

B Thigh

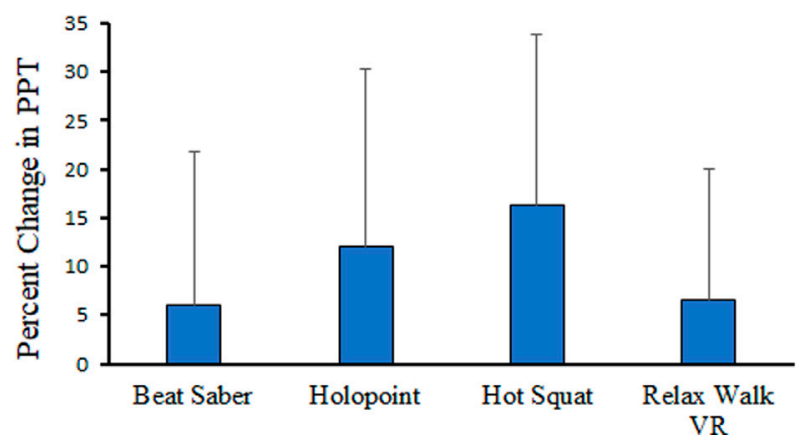

FIGURE 2 | Means and standard deviations (SD) for Percentage Change (\%) in Pressure Pain Thresholds at the forearm (A) and thigh (B) from Pre-VR to Post-VR across Game. 
TABLE 4 | Means and standard deviations (SD) for accelerometer variables (time spent [minutes]) for each game and body site.

\begin{tabular}{ccccc}
\hline & Beat Saber & Holopoint & Hot Squat & Relax Walk VR \\
\hline \multicolumn{7}{l}{$\begin{array}{l}\text { Physical activity of dominant upper arm (Wrist accelerometer) } \\
\text { Sedentary }\end{array}$} & $0.90(0.48)$ & $0.47(1.41)$ & $3.41(2.07)$ & $11.95(1.98)$ \\
MVPA & $10.05(1.94)$ & $10.75(2.46)$ & $5.57(2.63)$ & $0.52(1.08)$ \\
Whole-body physical activity (Waist accelerometer) & \\
Sedentary & $10.91(2.45)$ & $6.72(3.69)$ & $4.36(1.85)$ & $12.86(0.61)$ \\
MVPA & $0.28(0.27)$ & $2.04(2.24)$ & $4.51(2.56)$ & $0.03(0.09)$ \\
Physical activity & of the ipsilateral lower limb (Thigh accelerometer) & \\
Sedentary & $11.67(1.28)$ & $6.15(3.38)$ & $5.29(2.15)$ & $12.86(0.58)$ \\
MVPA & $0.16(0.17)$ & $1.89(2.50)$ & $3.55(2.47)$ & $0.03(0.10)$
\end{tabular}

MVPA, moderate-to-vigorous physical activity; VR, virtual reality.

change in PPT's was significantly higher for Hot Squat than for Beat Saber and Relax Walk. No other differences in percent change in PPT's among the VR games was found. Means and standard deviations for percent change in PPT's for each game by body site is in Figure 2.

\section{Physical Activity During VR Games Physical Activity of the Dominant Upper Limb (Arm Accelerometer)}

Time in Sedentary Time

The ANOVA showed a main effect for game $(p<0.001)$. The Tukey HSD test showed that arm sedentary time was significantly greater for Relax Walk compared to all other games. Hot Squat also had significantly greater arm sedentary time compared to Holopoint and Beat Saber (Table 4).

\section{Time in MVPA}

The ANOVA showed a main effect of game $(p<0.001)$. The Tukey HSD test showed that time spent in arm MVPA for Beat Saber and Holopoint was significantly higher than Hot Squat and Relax Walk. Hot Squat also elicited significantly higher arm MVPA than Relax Walk (Table 4).

\section{Whole Body Physical Activity (Waist Accelerometer) Time in Sedentary Time}

The ANOVA showed a main effect for game $(p<0.001)$. The Tukey HSD test showed significant differences between all games in whole body sedentary time. Whole body sedentary time was lowest for Hot Squat followed by Holopoint, and then Beat Saber, and lastly Relax Walk (game with highest sedentary time) (Table 4).

\section{Time in MVPA}

The ANOVA showed a main effect for game $(p<0.001)$. The Tukey HSD test showed that time spent in whole body MVPA for Hot Squat was significantly higher than all other games. Additionally, Holopoint elicited significantly higher whole body MVPA compared to Beat Saber and Relax Walk (Table 4).

\section{PA of the Dominant Lower Limb (Thigh Accelerometer) Time in Sedentary Time}

The ANOVA showed a main effect of game $(p<0.001)$. The Tukey HSD test showed that Beat Saber and Relax Walk had significantly higher lower-limb sedentary time than Holopoint and Hot Squat (Table 4).

\section{Time in MVPA}

The ANOVA showed a main effect of game $(p<0.001)$. The Tukey HSD test showed that Hot Squat elicited significantly higher lower-limb MVPA than all other games. Additionally, lower body MVPA for Holopoint was significantly higher than Beat Saber and Relax Walk (Table 4).

\section{Summary of Results}

Overall, the results showed a significant hypoalgesic effect in the forearm and thigh following acute bouts of VR gameplay. No significant differences were found between games for the PPT percent change scores of the forearms. However, the results revealed significant differences between games in PPT percent change scores for the thigh, with Hot Squat eliciting the largest hypoalgesic effect. The accelerometer results confirmed differences in physical activity between games, with Hot-Squat eliciting the highest level of lower- and whole-body MVPA and Relax Walk eliciting almost no physical activity.

\section{DISCUSSION}

The purpose of this study was to assess whether playing physically active VR games would have an acute hypoalgesic effect on experimentally induced pain in young, healthy adults, and whether this effect would differ between games eliciting varying degrees of physical activity. Evaluation of the physical activity data verified that Relax Walk was a non-active game. As expected, Hot Squat elicited the most lower and whole-body activity ( $4.5 \mathrm{~min}$ of MVPA and $4 \mathrm{~min}$ of light activity out of 13 recorded minutes). Additionally, Beat Saber and Holopoint elicited mostly arm MVPA ( 10 min), with only a little lower and whole-body movement. Importantly, prior active gaming research has shown that lower and whole-body movement compared to upper-limb movement is more important to reaching energy expenditure levels consistent with MVPA (Jordan et al., 2011; Duncan and Dick, 2012; Scheer et al., 2014).

Based on prior literature, the first hypothesis was that playing VR games, regardless of physical activity levels, would acutely decrease pressure pain sensitivity following each gaming session. Importantly, we first showed that PPTs did not change from the familiarization trials to the pretest trials, indicating that the PPTs likely did not change due to repeated pain testing. The pre- and post-test PPT data supported our first hypothesis as participants experienced an overall hypoalgesic effect in the forearm and thigh following $15 \mathrm{~min}$ bouts of VR gaming. Notably, the magnitude of the hypoalgesic effect on the forearm did not differ between VR games. Previous VR research has primarily focused on VR games or experiences that involve little to no physical activity. The research indicates that passively engaging in VR while seated or standing reduces pain sensitivity (Magora et al., 2006; Boylan et al., 2018). The primary mechanism attributed to these effects involve distraction. This distraction theory suggests that while focus is given to stimuli other than pain, the pain stimulus would 
not be perceived as painful. This is based on the understanding that sensory systems have limited capacity in focusing on multiple external stimuli simultaneously; therefore, the external stimuli would draw the attention of the individual from the pain stimulus (McCaul and Malott, 1984; Hoffman et al., 2007). The PPT data during Relax Walk from the current study particularly supports the prior literature showing that non-active VR games and experiences could be used as a distraction tool for pain (Magora et al., 2006; Hoffman et al., 2007).

We also hypothesized that playing VR games which required more physical activity or movement (i.e., Beat Saber, Holopoint, and Hot Squat) would have a greater hypoalgesic effect than the non-active VR game (Relax Walk). Prior research shows that bouts of moderate-intensity physical activity and non-active VR separately induce hypoalgesic effects (Naugle et al., 2012; Jin et al., 2016; Glennon et al., 2018). However, whether the hypoalgesic effects of VR could be enhanced by adding physical activity to the VR experience (i.e., VR that incorporates physical activity) has received little attention. Importantly, the data in the current study provided strong and novel evidence for an enhanced hypoalgesic effect when combining moderate-intensity whole-body movements and VR distraction compared to VR distraction alone. Specifically, the magnitude of pain reduction at the thigh was greatest for the game eliciting the greatest amount of whole and lower-body MVPA (i.e., Hot Squat). Indeed, the magnitude of pain reduction following Hot Squat was over twice as high as the game requiring minimal movement at any body site (Relax Walk) and the game with very little whole-body and thigh MVPA (Beat Saber). This is in accordance with prior active gaming research showing that a primary factor determining whether an active game (i.e., non-VR active games) elicits exercise-induced hypoalgesia is the intensity level reached during game play. (Carey et al., 2017; Naugle et al., 2017).

Prior research had provided preliminary evidence that hypoalgesia could be enhanced when the distraction effects of VR are combined with movement. For example, Czub, et al. (Czub and Piskorz, 2017) evaluated how different levels of arm movement using scaled computer mouse movements during VR gameplay affected cold pain sensitivity in healthy adults. The authors found that larger arm movements elicited lower pain intensities during cold water immersion than smaller arm movements, suggesting that more movement may be associated with reducing pain perception in VR tasks. Another study compared the hypoalgesic effects of VR combined with exercise imagery to VR distraction (Hayashi et al., 2019). The results revealed that VR combined with exercise imagery resulted in higher pressure pain thresholds during the VR task compared to VR without exercise imagery. However, the current study is the first to examine the impact of VR experiences that include actual light to moderate intensity whole body movements on pain sensitivity.

Contrary to expectations, an enhanced hypoalgesic effect of active VR compared to non-active VR was found only when measuring PPTs of the thigh vs. the forearm, although the forearm data trended in the hypothesized direction. Several explanations could account for the differing results observed on these two body parts. First, exercise-induced hypoalgesia can be produced by both local and central pain inhibitory effects and these effects may be stronger when both effects are combined. Local effects are characterized by reductions in pain in the active or exercising limb. Central effects are characterized by pain reductions in body parts distant to the exercising muscle (Gomolka et al., 2019). If the physical activity during VR gameplay induced central pain inhibitory effects, we would have expected a greater magnitude of pain reduction following Hot Squat compared to the other games at both the thigh and forearm. Central pain inhibitory effects following aerobic exercise usually requires the exercise to be at least moderate to vigorous intensity (Naugle et al., 2012; Micalos and Arendt-Nielsen, 2016; Vaegter et al., 2018), with greater effects evident at higher intensities. Thus, the active VR games possibly did not elicit intense enough physical activity or cardiovascular response to produce a central pain inhibitory response above and beyond that of the VR distraction. Further, the game requiring the most moderate to vigorous movement of the leg, Hot Squat, produced the greatest hypoalgesic effect on the leg compared to the other games. Thus, Hot Squat may have induced a stronger pain inhibitory effect on the thigh by combining both local and central mechanisms. These mechanisms could include changes in ß-endorphins, changes in plasma adrenaline and noradrenalin, peripheral nociceptive inhibition, and expressed endogenous opioid substances located both centrally and locally during and after exercise (Kosek and Lundberg, 2003; Tegeder et al., 2003; Naugle et al., 2012; Vaegter et al., 2014; Micalos and Arendt-Nielsen, 2016).

\section{Limitations and Future Work}

Several limitations within this study need to be addressed. First, the study sample included only healthy younger adults that reported high levels of physical activity; therefore, the results may not generalize to other populations. Future research should evaluate whether active VR games could have similar hypoalgesic effects in different populations, including but not limited to older adults, sedentary individuals, and those with chronic and acute pain conditions. Second, we used PPTs as the mode to evaluate pain sensitivity. Other methods are available to use for pain assessment, such as heat pain sensitivity via thermodes and cold-water immersion. Pressure pain testing was chosen in the current study because it utilized a portable pressure algometer, which made pain sensitivity testing more feasible to perform. Prior research has shown that hypoalgesic responses to exercise is partially a function of the experimental pain test (Naugle et al., 2013); thus, we may have found different results with other methods of experimentally induced pain. In addition, our study design focused on assessing whether playing physically activity VR games would have a greater hypoalgesic effect than the non-active VR game. As such, we did not include an exercise only condition. Thus, we do not know whether active VR induces an enhanced hypoalgesic effect compared to physical activity alone.

\section{CONCLUSION}

In conclusion, we added to the body of evidence demonstrating that VR elicits hypoalgesic effects and showed for the first time an 
enhanced hypoalgesic effect of physically active VR compared to non-active VR. Collectively, our results suggest that both nonactive and active VR should be explored as an alternative mode for pain management. Furthermore, deconditioned or sedentary individuals could still benefit from the hypoalgesic effects of nonactive VR engagement, as seen with Relax Walk. Moreover, future research should explore active VR gaming as a viable exercise option for those with pain conditions. Given the interactive nature of active VR games, these games could possibly serve as a pleasant distraction from pain symptoms in individuals with chronic pain and thereby enhance compliance with exercise therapy. However, future research needs to test this hypothesis.

\section{DATA AVAILABILITY STATEMENT}

The raw data supporting the conclusions of this article will be made available by the authors, without undue reservation.

\section{REFERENCES}

Aadland, E., and Ylvisåker, E. (2015). Reliability of the Actigraph GT3X+ Accelerometer in Adults under Free-Living Conditions. PLoS One 10 (8), e0134606. doi:10.1371/journal.pone.0134606

Aytar, A., Senbursa, G., Baltaci, G., Yuruk, Z. O., and Pekyavas, N. O. (2014). Reliability of Pressure Pain Thresholds in Healthy Young Adults. J. Musculoskelet. Pain 22 (3), 225-231. doi:10.3109/10582452.2014.883033

Bisset, L. M., Evans, K., and Tuttle, N. (2015). Reliability of 2 Protocols for Assessing Pressure Pain Threshold in Healthy Young Adults. J. Manipulative Physiol. Ther. 38 (4), 282-287. doi:10.1016/j.jmpt.2015.03.001

Boylan, P., Kirwan, G. H., and Rooney, B. (2018). Self-reported Discomfort when Using Commercially Targeted Virtual Reality Equipment in Discomfort Distraction. Virtual Reality 22, 309-314. doi:10.1007/s10055-017-0329-9

Carey, C., Naugle, K. E., Aqeel, D., Ohlman, T., and Naugle, K. M. (2017). Active Gaming as a Form of Exercise to Induce Hypoalgesia. Games Health J. 6 (4), 255-261. doi:10.1089/g4h.2017.0024

Chesterton, L. S., Sim, J., Wright, C. C., and Foster, N. E. (2007). Interrater Reliability of Algometry in Measuring Pressure Pain Thresholds in Healthy Humans, Using Multiple Raters. Clin. J. Pain 23, 760-766. doi:10.1097/ ajp.0b013e318154b6ae

Craig, C. L., Marshall, A. L., Sjostrom, M., Bauman, A. E., Booth, M. L., Ainsworth, B. E., et al. (2003). International Physical Activity Questionnaire: 12-Country Reliability and Validity. Med. Sci. Sports. Exerc. 35 (8), 1381-1395.

Czub, M., and Piskorz, J. (2014). "How Body Movement Influences Virtual Reality Analgesia," in Interactive Technologies and Games (iTAG), 2014 International Conference, Health, Disability and EducationAt, Nottingham, United kingdom. doi:10.1109/iTAG.2014.8

Czub, M., and Piskorz, J. (2017). Body Movement Reduces Pain Intensity in Virtual Reality-Based Analgesia. Int. J. Human-Computer Interaction 34 (11), 1045-1051. doi:10.1080/10447318.2017.1412144

Demeter, N., Josman, N., Eisenberg, E., and Pud, D. (2015). Who Can Benefit from Virtual Reality to Reduce Experimental Pain? A Crossover Study in Healthy Subjects. Eur. J. Pain 19 (10), 1467-1475. doi:10.1002/ejp.678

Duncan, M., and Dick, S. (2012). Energy Expenditure and Enjoyment of Exergaming: A Comparison of the Nintendo Wii and the Gamercize Power Stepper in Young Adults. Med. Sport 16 (3), 92-98. doi:10.5604/17342260.1011386

Evans, E., Naugle, K. E., Kaleth, A. S., Arnold, B., and Naugle, K. M. (2021). Physical Activity Intensity, Perceived Exertion, and Enjoyment during HeadMounted Display Virtual Reality Games. Games Health J. 10, 314-320. doi:10.1089/g4h.2021.0036

Freedson, P. S., Melanson, E., and Sirard, J. (1998). Calibration of the Computer Science and Applications, Inc. Accelerometer. Med. Sci. Sports Exerc. 30 (5), 777-781. doi:10.1097/00005768-199805000-00021

\section{ETHICS STATEMENT}

The studies involving human participants were reviewed and approved by the Indiana University Institutional Review Board (IRB). The patients/participants provided their written informed consent to participate in this study.

\section{AUTHOR CONTRIBUTIONS}

EE, KMN, KEN, BA, AK contributed to study design. Data collection was performed by EE and AO. Data analyses were performed by EE under the supervision of KMN. Data interpretation was performed by EE under the supervision of KMN, KEN, AK, BA. EE drafted the manuscript, KMN revised the manuscript. AK, BA, KEN, and AO provided critical revisions. All authors approved the final version of the manuscript for submission.

Glennon, C., McElroy, S., Connelly, L., Mische Lawson, L., Bretches, A., Gard, A., et al. (2018). Use of Virtual Reality to Distract from Pain and Anxiety. Onf 45 (4), 545-552. doi:10.1188/18.onf.545-552

Gomolka, S., Vaegter, H. B., Nijs, J., Meeus, M., Gajsar, H., Hasenbring, M. I., et al. (2019). Assessing Endogenous Pain Inhibition: Test-Retest Reliability of Exercise-Induced Hypoalgesia in Local and Remote Body Parts after Aerobic Cycling. Pain Med. 20, 2272-2282. doi:10.1093/pm/pnz131

Hayashi, K., Aono, S., Shiro, Y., and Ushida, T. (2019). Effects of Virtual RealityBased Exercise Imagery on Pain in Healthy Individuals. Biomed. Res. Int. 2019, 5021914. doi:10.1155/2019/5021914

Hoffman, H. G., Doctor, J. N., Patterson, D. R., Carrougher, G. J., and Furness, T. A., 3rd (2000). Virtual Reality as an Adjunctive Pain Control during Burn Wound Care in Adolescent Patients. Pain 85 (1-2), 305-309. doi:10.1016/ s0304-3959(99)00275-4

Hoffman, H. G., Garcia-Palacios, A., Kapa, V., Beecher, J., and Sharar, S. R. (2003). Immersive Virtual Reality for Reducing Experimental Ischemic Pain. Int. J. HumanComputer Interaction 15 (3), 469-486. doi:10.1207/s15327590ijhc1503_10

Hoffman, H. G., Patterson, D. R., and Carrougher, G. J. (2000). Use of Virtual Reality for Adjunctive Treatment of Adult Burn Pain during Physical Therapy: a Controlled Study. The Clin. J. Pain 16 (3), 244-250. doi:10.1097/00002508200009000-00010

Hoffman, H. G., Richards, T. L., Van Oostrom, T., Coda, B. A., Jensen, M. P., Blough, D. K., et al. (2007). The Analgesic Effects of Opioids and Immersive Virtual Reality Distraction: Evidence from Subjective and Functional Brain Imaging Assessments. Anesth. Analg 105 (6), 1776-1783. doi:10.1213/ 01.ane.0000270205.45146.db

Jin, W., Choo, A., Gromala, D., Shaw, C., and Squire, P. (2016). A Virtual Reality Game for Chronic Pain Management: A Randomized, Controlled Clinical Study. Stud. Health Technol. Inform. 220, 154-160.

Jones, D., Crossley, K., Dascombe, B., Hart, H. F., and Kemp, J. (2018). Validity and Reliability of the Fitbit Flex and Actigraph Gt3X+ at Jogging and Running Speeds. Intl J. Sports Phys. Ther. 13 (5), 860-870. doi:10.26603/ijspt20180860

Jordan, M., Donne, B., and Fletcher, D. (2011). Only Lower Limb Controlled Interactive Computer Gaming Enables an Effective Increase in Energy Expenditure. Eur. J. Appl. Physiol. 111 (7), 1465-1472. doi:10.1007/s00421-010-1773-3

Kelly, L. A., McMillan, D. G., Anderson, A., Fippinger, M., Fillerup, G., and Rider, J. (2013). Validity of Actigraphs Uniaxial and Triaxial Accelerometers for Assessment of Physical Activity in Adults in Laboratory Conditions. BMC Med. Phys. 13 (5), 5. doi:10.1186/1756-6649-13-5

Kemppainen, P., Paalasmaa, P., Pertovaara, A., Alila, A., and Johansson, G. (1990). Dexamethasone Attenuates Exercise-Induced Dental Analgesia in Man. Brain Res. 519, 329-332. doi:10.1016/0006-8993(90)90096-t

Kim, Y., Barry, V. W., and Kang, M. (2015). Validation of the ActiGraph GT3X and activPAL Accelerometers for the Assessment of Sedentary Behavior. Meas. Phys. Edu. Exerc. Sci. 19 (3), 125-137. doi:10.1080/1091367x.2015.1054390 
Koltyn, K. F. (2000). Analgesia Following Exercise. Sports Med. 29, 85-98. doi:10.2165/00007256-200029020-00002

Kosek, E., and Lundberg, L. (2003). Segmental and Plurisegmental Modulation of Pressure Pain Thresholds during Static Muscle Contractions in Healthy Individuals. Eur. J. Pain 7 (3), 251-258. doi:10.1016/s1090-3801(02)00124-6

Law, E. F., Dahlquist, L. M., Sil, S., Weiss, K. E., Herbert, L. J., Wohlheiter, K., et al. (2011). Videogame Distraction Using Virtual Reality Technology for Children Experiencing Cold Pressor Pain: the Role of Cognitive Processing. J. Pediatr. Psychol. 36 (1), 84-94. doi:10.1093/jpepsy/jsq063

Lotze, M., and Halsband, U. (2006). Motor Imagery. J. Physiol. Paris 99 (4-6), 386-395. doi:10.1016/j.jphysparis.2006.03.012

Magora, F., Cohen, S., Shochina, M., and Dayan, E. (2006). Virtual Reality Immersion Method of Distraction to Control Experimental Ischemic Pain. Isr. Med. Assoc. J. 8, 261-265.

McCaul, K. D., and Malott, J. M. (1984). Distraction and Coping with Pain. Psychol. Bull. 95 (3), 516-533. doi:10.1037/0033-2909.95.3.516

Micalos, P. S., and Arendt-Nielsen, L. (2016). Differential Pain Response at Local and Remote Muscle Sites Following Aerobic Cycling Exercise at Mild and Moderate Intensity. Springerplus 5, 91. doi:10.1186/s40064-016-1721-8

Miller, K. J., Schalk, G., Fetz, E. E., den Nijs, M., Ojemann, J. G., and Rao, R. P. N. (2010). Cortical Activity during Motor Execution, Motor Imagery, and Imagery-Based Online Feedback. Proc. Natl. Acad. Sci. 107 (9), 4430-4435. doi:10.1073/pnas.0913697107

Naugle, Carey. C., Ohlman, T., Godza, M., Mikesky, A., and Naugle, K. M. (2019). Improving Active Gaming's Energy Expenditure in Health Adults Using Structured Playing Instructions for the Nintendo Wii and Xbox Kinect. J. Strength Conditioning Res. 33, 549-558. doi:10.1519/JSC.0000000000002997

Naugle, K. E., Parr, J. J., Chang, S., and Naugle, K. M. (2017). Active Gaming as Pain Relief Following Induced Muscle Soreness in a College-Aged Population. Athletic Train. Sports Health Care 9 (5), 225-232. doi:10.3928/19425864-20170619-03

Naugle, K. M., Naugle, K. E., Fillingim, R. B., and Riley, J. L., 3rd (2013). Isometric Exercise as a Test of Pain Modulation: Effects of Experimental Pain Test, Psychological Variables, and Sex. Pain Med. 15, 692-701. doi:10.1111/pme.12312

Naugle, K. M., Fillingim, R. B., and Riley, J. L., 3rd (2012). A Meta-Analytic Review of the Hypoalgesic Effects of Exercise. The J. Pain 13 (12), 1139-1150. doi:10.1016/j.jpain.2012.09.006

Naugle, K. M., Naugle, K. E., Fillingim, R. B., Samuels, B., and Riley, J. L., 3rd (2014). Intensity Thresholds for Aerobic Exercise-Induced Hypoalgesia. Med. Sci. Sports Exerc. 46 (4), 817-825. doi:10.1249/mss.0000000000000143

Naugle, K. M., Naugle, K. E., and Riley, J. L., 3rd (2016). Reduced Modulation of Pain in Older Adults after Isometric and Aerobic Exercise. J. Pain 17 (6), 719-728. doi:10.1016/j.jpain.2016.02.013
Rice, D., Nijs, J., Kosek, E., Wideman, T., Hasenbring, M. I., Koltyn, K., et al. (2019). Exercise-Induced Hypoalgesia in Pain-free and Chronic Pain Populations: State of the Art and Future Directions. J. Pain 20, 1249-1266. doi:10.1016/ j.jpain.2019.03.005

Scheer, K. S., Siebrant, S. M., Brown, G. A., Shaw, B. S., and Shaw, I. (2014). Wii, Kinect, and Move. Heart Rate, Oxygen Consumption, Energy Expenditure, and Ventilation Due to Different Physically Active Video Game Systems in College Students. Int. J. Exerc. Sci. 7 (1), 22-32.

Tegeder, I., Meier, S., Burian, M., Schmidt, H., Geisslinger, G., and Lötsch, J. (2003). Peripheral Opioid Analgesia in Experimental Human Pain Models. Brain 126 (Pt 5), 1092-1102. doi:10.1093/brain/awg115

Vaegter, H. B., Dørge, D. B., Schmidt, K. S., Jensen, A. H., and Graven-Nielsen, T. (2018). Test-Retest Reliabilty of Exercise-Induced Hypoalgesia after Aerobic Exercise. Pain Med. 19 (11), 2212-2222. doi:10.1093/pm/pny009

Vaegter, H. B., Handberg, G., and Graven-Nielsen, T. (2014). Similarities between Exercise-Induced Hypoalgesia and Conditioned Pain Modulation in Humans. Pain 155 (1), 158-167. doi:10.1016/j.pain.2013.09.023

Waller, R., Straker, L., O'Sullivan, P., Sterling, M., and Smith, A. (2015). Reliability of Pressure Pain Threshold Testing in Healthy Pain Free Young Adults. Scand. J. Pain 9 (1), 38-41. doi:10.1016/j.sjpain.2015.05.004

Weaver, J. B., 3rd, Mays, D., Sargent Weaver, S., Kannenberg, W., Hopkins, G. L., Eroĝlu, D., et al. (2009). Health-risk Correlates of Video-Game Playing Among Adults. Am. J. Prev. Med. 37 (4), 299-305. doi:10.1016/ j.amepre.2009.06.014

Conflict of Interest: The authors declare that the research was conducted in the absence of any commercial or financial relationships that could be construed as a potential conflict of interest.

Publisher's Note: All claims expressed in this article are solely those of the authors and do not necessarily represent those of their affiliated organizations, or those of the publisher, the editors and the reviewers. Any product that may be evaluated in this article, or claim that may be made by its manufacturer, is not guaranteed or endorsed by the publisher.

Copyright (C) 2021 Evans, Naugle, Ovispo, Kaleth, Arnold and Naugle. This is an open-access article distributed under the terms of the Creative Commons Attribution License (CC BY). The use, distribution or reproduction in other forums is permitted, provided the original author(s) and the copyright owner(s) are credited and that the original publication in this journal is cited, in accordance with accepted academic practice. No use, distribution or reproduction is permitted which does not comply with these terms. 